\title{
Cardiotoxicity of Contemporary Anticancer Immunotherapy
}

Natalie Dalbo, $M D^{1}$

Rushin Patel, $M D^{2}$

Rohan Parikh, $M D^{2}$

Sachin P. Shah, $M D^{2}$

Avirup Guha, $M D^{3}$

Sourbha S. Dani, $M D^{2}$

Sarju Ganatra, $M D^{4, *}$

\author{
Address \\ ${ }^{1}$ Division of Internal Medicine, Department of Medicine, Lahey Hospital and \\ Medical Center, Burlington, MA, USA \\ ${ }^{2}$ Division of Cardiovascular Medicine, Department of Medicine, Lahey Hospital and \\ Medical Center, Burlington, MA, USA \\ ${ }^{3}$ Harrington Heart and Vascular Institute, Case Western Reserve University School \\ of Medicine, Cleveland, USA \\ ${ }^{*}, 4$ Cardio-Oncology Program, Division of Cardiovascular Medicine, Department of \\ Medicine, Lahey Hospital and Medical Center, 41 Mall Road, Burlington, MA, \\ 01805, USA \\ Email: Sarju.ganatra@lahey.org
}

Published online: 3 November 2020

(C) Springer Science+Business Media, LLC, part of Springer Nature 2020

This article is part of the Topical Collection on Cardio-oncology

Keywords Immunotherapy - Immune checkpoint inhibitor - ICI • CAR T cell therapy - Chimeric antigen receptor • Cardiotoxicity · Cardio-oncology

\section{Abstract}

Purpose of review Contemporary anticancer immunotherapy, particularly immune checkpoint inhibitors (ICI) and chimeric antigen receptor (CAR) T cell therapy, has changed the landscape of treatment for patients with a variety of malignancies who historically had a poor prognosis. However, both immune checkpoint inhibitors and CAR T cell therapy are associated with serious cardiovascular adverse effects. As immunotherapy evolves to include high-risk patients with preexisting cardiovascular risk factors and disease, the risk and relevance of its associated cardiotoxicity will be even higher.

Recent findings ICI can cause myocarditis, which usually occurs early after initiation, can be fulminant, and prompt treatment with high-dose corticosteroids is crucial. CAR T cell therapy frequently leads to cytokine release syndrome, which is associated with 
cardiomyopathy or arrhythmia development and may also result in circulatory collapse. Supportive treatment, as well as tocilizumab, an anti-interleukin- 6 receptor antibody, is the cornerstone of treatment. Recent findings suggest that preexisting cardiovascular risk factors and disease may increase the risk of such cardiotoxicity, and prompt recognition, as well as treatment, may favorably alter the outcomes.

Summary ICI and CAR T cell therapy have improved cancer-related outcomes; however, they both are associated with potentially therapy-limiting cardiotoxicity. Cardiooncologists are required to play an important role in patient selection, pretherapy cardiovascular optimization, and prompt recognition and treatment of cardiotoxicity.

\section{Introduction}

Contemporary immunotherapy, such as immune checkpoint inhibitors (ICI) and chimeric antigen receptor (CAR) T cell therapy, has dramatically improved outcomes for a wide variety of cancers with an otherwise poor prognosis [1-5]. However, both of these therapies can potentially lead to serious cardiovascular adverse effects $[6,7,8 \bullet \bullet]$. As the use of immunotherapy expands for the treatment of patients with a wide variety of malignancies and preexisting cardiovascular disease or risk factors, such cardiotoxicity will be observed more frequently. Hence, the strategies for pretherapy risk stratification as well as surveillance to enable prompt recognition and treatment of cardiotoxicity are increasingly important. A multidisciplinary approach is crucial for the management of patients on novel immunotherapies, and cardio-oncologists will play a fundamental role in the comprehensive care of these patients.

Immune checkpoint inhibitors (ICI) work by releasing restrained antitumor immune responses. To date, seven ICIs have been approved by the Food and Drug Administration (FDA) for 12 different cancers [6, 9]. While these agents have revolutionized the outcomes for a wide variety of cancers, high-grade immune-related adverse events (irAEs) can occur, particularly with combination immunotherapy $[10 \bullet \bullet]$. While many irAEs are treated with temporary cessation of ICI therapy and immunosuppression, cardiotoxicity in the form of myocarditis is potentially therapy-limiting adverse events with a high rate of associated significant morbidity and mortality $[10 \bullet \bullet]$.

Chimeric antigen receptor (CAR) T cell therapy has shifted a paradigm for the treatment of patients with relapsed and refractory hematologic malignancies [35]. It is also being investigated as a therapeutic option of other malignancies. Moreover, there is growing enthusiasm to explore the utility of genetically engineered $\mathrm{T}$ cells in the treatment of autoimmune disease and infections [11]. However, CAR T cell therapy is associated with potentially life-threatening cytokine release syndrome (CRS), which in turn may lead to arrhythmia, myocardial injury, cardiomyopathy, and cardiovascular collapse $[8 \bullet \bullet, 12]$. Although insults related to CRS toxicity might be transient and reversible in most instances in patients with adequate cardiovascular reserve, they can be particularly challenging in higher risk, often elderly, patients with preexisting cardiovascular disease.

Here, we review the cardiotoxicity associated with ICI and CAR T cell therapy and discuss the management strategies.

\section{Immune checkpoint inhibitors}

ICIs enhance antitumor immunity by blocking intrinsic downregulators of immunity, such as cytotoxic T lymphocyte antigen 4 (CTLA-4) and programmed cell death 1 (PD-1) or its ligand, programmed cell death ligand 1 (PD-L1). To date, the US Food and Drug Administration (FDA) has approved seven agents (one CTLA-4-blocking antibody (ipilimumab), three PD-1- 
blocking antibodies (nivolumab, pembrolizumab, and cemiplimab), and three PD-L1-blocking antibodies (atezolizumab, avelumab, and durvalumab)) for 12 different cancers $[6,9]$. Many more agents are under investigation for the treatment of a wide variety of cancers.

\section{Cardiotoxicity}

As ICIs unrestraint the innate immune system nonselectively, they are associated with several irAEs involving various organ systems such as the gastrointestinal tract, endocrine glands, skin, and liver [9]. Myocarditis is less frequent than other irAEs. While the fulminant form of myocarditis is reported more frequently due to associated major morbidity and high mortality, smoldering myocarditis, as well as other forms of cardiotoxicity such as cardiomyopathy, arrhythmia, and vasculitis, has also been reported [6, 7].

The mechanism of ICI-associated cardiotoxicity is not well understood. However, common high-frequency $\mathrm{T}$ cell receptor sequences have been noted in the tumor and cardiac muscle, which raises the possibility of a shared antigen target [13]. All three targets of ICIs-CTLA-4, PD-1, and PD-L1-have shown cardioprotective effects in animal studies, and their inhibition may potentially be implicated for the occurrence of myocarditis [14-17]. However, ICIassociated myocarditis occurs only in selective patients, and hence, other potential mechanisms or risk factors ought to play an important role.

ICI-associated myocarditis may present in varied ways, including mild, nonspecific symptoms, such as fatigue and myalgia, to more specific and severe symptoms such as chest pain, and shortness of breath to syncope and sudden cardiac death. In an international multicenter registry, nearly half of the patients with myocarditis developed major adverse cardiovascular effects, including atrial and ventricular arrhythmias, complete heart block, heart failure, cardiogenic shock, or death. Although fulminant myocarditis with heart failure and arrhythmias has been more commonly reported, subclinical or smoldering myocarditis with minimal signs and symptoms may also occur.

\section{Timing of Cardiotoxicity}

Typically, ICI-associated myocarditis occurs early after the initiation of therapy within the median time of 2 months, with most of the cases occurring within

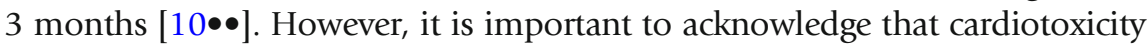
may occur at any time during or even after the cessation of therapy due to persistent immune effects [18]. underestimated due to a combination of factors including the lack of specificity in the clinical presentation, the potential of overlap with other cardiovascular and general medical illnesses, the challenges in the diagnosis, and a general lack of awareness of this condition. The overall prevalence of ICI-associated myocarditis was reported $1.14 \%$, which increased to as high as $2.4 \%$ for combination therapy with anti-PD-1/anti-CTLA-4 agents in an international multicenter 
registry $[10 \bullet \bullet]$. For patients undergoing nivolumab therapy, troponin level was found to be elevated in $10 \%$ of patients without a clear cause, which suggests that the true incidence of subclinical or smoldering myocarditis might be even higher [19].

While the risk factors for ICI-associated myocarditis are not well characterized, a combination therapy with two or more ICIs, anti-CTLA-4 therapy, diabetes, and

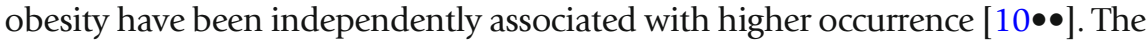
prevalence of myocarditis is reportedly lowest with the anti-PD-1 agent $(0.5 \%)$, whereas it is higher with other agents: $2.4 \%$ with anti-PD-L1 and $3.3 \%$ with

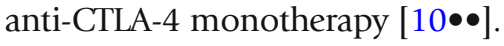

In general, noncardiac irAEs are more frequently reported in patients with preexisting autoimmune disease. However, as far as myocarditis is concerned, no such association with preexisting autoimmune disease has been established [6]. Similarly, while in a small study, influenza vaccination was associated with a higher rate of irAEs [20], in an international multicenter registry, the rate of influenza vaccination among ICI-related myocarditis cases was lower than the controls on ICIs who did not develop myocarditis, rejecting any such association of influenza vaccination with development of immune-mediated myocarditis. On the contrary, among those who developed ICI-associated myocarditis, the degree of myocardial injury and the risk of MACE were lower in patients who had received an influenza vaccine [21].

Unpublished data from the multicenter international registry also demonstrates that those who developed myocarditis were more likely to be male $(65 \%$ vs. $55 \%, p=0.02)$ and had a higher rate of hypertension $(60 \%$ vs. $48 \%, p<0.009)$ as well as rates of smoking ( $48 \%$ vs. $17 \%$, $p<0.001)$. Patients who developed myocarditis were also more likely to be on a statin (395 vs. $29 \%, p=0.04$ ) and angiotensin-converting enzyme inhibitor or aldosterone receptor blocker $(32 \%$ vs. $23 \%, p=0.04)$. This may suggest that preexisting cardiovascular risk factors are associated with the development of ICI-associated myocarditis. However, it is important to note that this interpretation is based upon proportional analysis rather than multivariate regression analysis, and hence, the true impact of each of these factors is still under investigation. Additionally, the risk of myocarditis was not found to be higher in patients with preexisting coronary artery disease, atrial fibrillation, or chronic kidney disease [22].

There are no specific guidelines for the diagnosis of ICI-associated myocarditis. However, given that the presentation of ICI-induced myocarditis can be nonspecific, a high degree of suspicion is required given that rapid, fulminant progression is possible. While it is reasonable to suspect myocarditis in any patient on ICI presenting with cardiovascular or other suggestive symptoms, the appropriate workup for other potential etiologies such as pneumonitis, other irAEs, viral myocarditis, or other common cardiovascular diseases such as acute coronary syndrome should be considered especially if workup for myocarditis is unrevealing. 
While there are several different cardiovascular tests which could be useful, there is no single best test for the definitive diagnosis of ICI-associated myocarditis. Our understanding regarding the application of these cardiovascular tests to diagnose ICI-associated myocarditis is rapidly evolving.

Electrocardiogram (ECG)

It is readily available and usually the first test performed on patients with cardiovascular symptoms. While ECG abnormality is reported in the majority of patients presenting with ICI-associated myocarditis, a normal ECG does not rule out myocarditis $[10 \bullet \bullet]$. Most often, the findings are nonspecific and may demonstrate sinus tachycardia, QRS/QT prolongation, conduction abnormalities, diffuse T wave inversion, abnormal Q wave, atrial or ventricular arrhythmia, and local or diffuse ST elevation. In a retrospective study, QRS prolongation was noted more frequently in patients with myocarditis as compared to controls, and it was also associated with a higher occurrence of MACE [6]. This finding signifies that any subtle new conduction abnormality should not be overlooked even in relatively asymptomatic or minimally symptomatic patients.

While troponin is shown to be one of the most sensitive tests, with increased troponin level noted in $94 \%$ of patients with ICI-associated myocarditis $[10 \bullet \bullet$, it is not specific for myocarditis, and a normal troponin level, especially those who present late after initiation of ICIs, does not exclude ICI-associated myocarditis. Troponin level, on the other hand, if elevated, may help with prognostication in such patients, with a higher

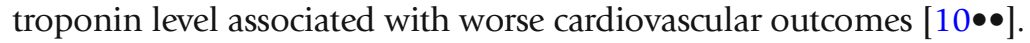

Brain natriuretic peptide (BNP)/N-terminal pro-BNP

These peptides are markers of myocardial stretch, and levels can be elevated in patients with volume overload/heart failure. However, they are neither sensitive nor specific enough to help with the diagnosis making in isolation $[7,10 \bullet \bullet$.

This is usually the first-line imaging test for the assessment of patients with suspected ICI-associated myocarditis. While some patients with myocarditis may develop cardiomyopathy, the left ventricular ejection fraction (LVEF) may be normal even in fulminant myocarditis [10••]. Importantly, a normal LVEF does not exclude the occurrence of a major adverse cardiac event (MACE). In an international retrospective registry, 51\% of patients with myocarditis had normal LVEF, and of those who experienced MACE, $38 \%$ occurred in patients with a normal $\operatorname{LVEF}[10 \bullet \bullet, 23]$. On the other side, emerging data show that global longitudinal strain (GLS) obtained during 2-D echocardiography may help with both the diagnosis and prognosis. A retrospective study of 101 patients with ICI-associated myocarditis shows 
that GLS was noted to be lower in patients with myocarditis compared with controls, for patients with both a preserved and a reduced ejection fraction. A lower GLS in this retrospective registry was strongly associated with subsequent MACE among patients with myocarditis regardless of whether they had preserved or reduced ejection fraction [23].

CMR is considered the gold standard noninvasive test for the diagnosis of myocarditis because of its excellent spatial resolution and additive ability to provide tissue characterization [24]. However, it may not be readily available, and severely ill patients may not be able to undergo a lengthy test, which limits its widespread utility for patients with suspected myocarditis. Additionally, recent data from an international registry show that the majority of patients with ICI-associated myocarditis had normal LVEF on CMR and only $46 \%$ had late gadolinium enhancement (LGE), which makes the current CMR diagnostic criteria a subpar tool for diagnosis of ICIassociated myocarditis. Although LGE on CMR has been shown to be an effective tool for risk stratification and prognostication for myocarditis of other causes, for ICI-associated myocarditis, LGE was not associated with MACE [25]. These data suggest that the LGE-only approach to diagnosing ICI-associated myocarditis is likely not adequate and additional parameters of tissue characterization may need to be routinely utilized in the evaluation of these patients.

While EMB is highly specific for the diagnosis of myocarditis, the myocardial involvement with ICI-associated myocarditis is usually patchy, which makes EMB less sensitive [26]. Additionally, given its invasive nature and the risk of cardiac perforation, with limited sensitivity, it is not usually performed as a first-line test. However, it certainly has a role in patients with otherwise unrevealing workup but with a high index of suspicion for ICIassociated myocarditis. A biopsy from the affected area typically shows inflammatory infiltrates (usually T cell-predominant lymphocytic infiltrate) in the myocardium. Immune-mediated myocarditis may also demonstrate the presence of cell-specific markers such as Tlymphocytes (CD3), macrophages (CD68), or human leukocyte antigens [7, 27].

Our know-how regarding the treatment of ICI-associated myocarditis is primarily based on clinical experience and retrospective registry data rather than largescale prospective trials. For any patients receiving ICI presenting with new or worsening cardiovascular symptoms, ICI-associated myocarditis should be suspected, any further ICI therapy should be withheld, and appropriate workup needs to be promptly performed. Depending on the degree of suspicion and clinical presentation, the patient may need to be hospitalized, particularly if the patient is unstable or have any significant abnormality on cardiovascular testing, including any subtle new conduction abnormalities on ECG given the potential fulminant and rapidly progressive nature of this condition. Prompt 
initiation of immunosuppressive therapy is crucial for the management of ICIassociated myocarditis.

A high dosage of corticosteroids, typically methylprednisolone $1000 \mathrm{mg} /$ day for 3 days, followed by prednisone $1 \mathrm{mg} / \mathrm{kg}$ is considered the first-line therapy [7]. An international registry data of 126 patients showed that higher initial dose and prompt initiation (within $24 \mathrm{~h}$ of presentation) of

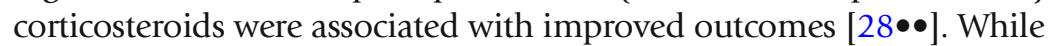
both dosing and timing were important, the initiation time of corticosteroids appeared to play a stronger role, such that using high-dose corticosteroids could not overcome the effect of corticosteroids given later. Contrary to that, lower dose corticosteroids administered early still was associated with a better outcome as compared to patients who receive high-dose corticosteroids later [ $28 \bullet \bullet$. This emphasizes the need for prompt initiation of corticosteroids, even prior to confirmation of the diagnosis. The optimal duration of treatment with corticosteroids is not well studied and needs to be individualized. However, most experts believe that it should be tapered slowly only after the resolution of symptoms and normalization of LVEF or stabilization of arrhythmia [7].

Beyond corticosteroids, the utility of other immunosuppressive agents is not well established. For stable patients with immune-mediated myocarditis, infliximab, tacrolimus, or mycophenolate mofetil might be considered if there is evidence of high-grade myocarditis on biopsy, for those who fail to respond to corticosteroid therapy, or as a steroid-sparing agent [6, 7]. However, the evidence regarding their use is limited and mostly is based on their use in patients with cardiac allograft rejection. Additionally, the use of infliximab has been associated with the development of heart failure [29], and hence, it may not be a suitable agent for patients with cardiomyopathy or heart failure. If the patient is unstable, antithymocyte globulin, intravenous immunoglobulin, and plasma exchange should be considered. Abatacept, a CTLA-4 agonist, and alemtuzumab, a CD52 monoclonal antibody, have shown to be effective in the treatment of corticosteroidrefractory ICI-associated myocarditis [30, 31]. The evidence regarding their utility is limited and based primarily on case reports. These agents could be considered in the management of patients with corticosteroid-refractory ICI-associated myocarditis.

For hemodynamically unstable patients with acute ICI-associated myocarditis, mechanical circulatory support may need to be considered. The data regarding its efficacy and safety in this population is not well studied, and hence, multidisciplinary discussion is crucial in such decision making after considering overall prognosis from cancer standpoint. For patients with cardiomyopathy, guideline-directed medical therapy should be initiated 
after stabilization. Similarly, for patients who develop atrial or ventricular arrhythmia, appropriate antiarrhythmic and anticoagulation therapy should be considered.

ICI-associated myocarditis is reportedly associated with very high mortality (38 to $46 \%$ ) and nonfatal MACE in nearly half of all myocarditis cases, including heart failure, cardiogenic shock, cardiac arrest, ventricular arrhythmia, and complete heart block $[10 \bullet \bullet, 32]$. It is possible that the rate of such complications is overestimated due to the lack of systemic screening and surveillance, which may lead to reporting and detection bias leading to underreporting of subclinical or smoldering myocarditis with minimal signs and symptoms.

ICI-associated myocarditis is considered high-grade toxicity, and the current guidelines recommend to permanently discontinue ICI therapy [33]. However, for patients with suspected myocarditis, with the milder course and complete recovery as well as with cancer progression, such a decision may need to be reconsidered. The decision to rechallenge a patient who has suffered ICIassociated myocarditis must be individualized and discussed among a multidisciplinary team. Variables to consider include cancer status, the severity of cardiotoxicity, regression of toxicity with immunosuppressive therapy, and patient preference. If the decision is made to rechallenge, monotherapy with an alternative agent and very close cardiovascular monitoring with cardiac imaging as well as troponin surveillance should be considered [6]. While there is no data regarding the rechallenge after myocarditis, retrospective analysis has shown that the anti-PD-1 agent was safely resumed after initially experiencing irAE with an anti-CTLA-4 agent [34].

\section{Chimeric antigen receptor T cell therapy}

Chimeric antigen receptor (CAR) T cells are genetically engineered $\mathrm{T}$ cells that express an artificial receptor capable of binding to tumor-associated antigens and thus augmenting $\mathrm{T}$ cell function [35]. In 2017, the FDA approved two agents that express CD19 on their surface, tisagenlecleucel, and axicabtagene ciloleucel. These agents are approved for limited indications currently: for children and young adults up to 25 years of age with relapsed or refractory B cell precursor acute lymphocytic leukemia (ALL) and large B cell lymphoma relapsed/refractory after two or more lines of systemic therapy including diffuse large B cell lymphoma (DLBCL) [3-5, 36]. Preliminary results from multiple phase I clinical trials of CAR T cells targeting B cell maturation antigen have shown encouraging results in relapsed or refractory multiple myeloma [37].

Cytokine release syndrome (CRS) is a frequent adverse effect of CAR T cell therapy, which consists of a constellation of signs and symptoms caused by supraphysiologic levels of inflammatory cytokines released by the activated CAR T cells and other immune cells such as macrophages [ $8 \bullet \bullet]$. Cardiotoxicity in the form of myocardial injury, as evident by troponin elevation, 
cardiomyopathy or heart failure development, arrhythmia, capillary leak syndrome, and circulatory collapse, are mostly reported in the context of highgrade CRS [38]. The pathophysiology of cardiac dysfunction during CRS is unclear but resembles cardiomyopathy associated with sepsis and stress, likely associated with interleukin-6 (IL-6), which has been implicated as a mediator of myocardial depression in infectious and inflammatory states [39]. Direct cardiotoxicity possibly attributed to autoimmunity with off-target cross-reactivity of $\mathrm{T}$ cells against Titin, a striated muscle protein in the heart, has been reported in two patients after the infusion of T cells targeting melanoma antigen family A3 (MAGE-A3) [40].

CRS usually develops early on after CAR T cell infusion (median time 2.2 days). While the highest risk is in the first 2 weeks after infusion but at least in theory, it could occur as long as CAR T cells persist in the circulation, which could be years [41]. Our study finds that cardiomyopathy developed after a median duration of 12.5 (range 2-24) days from CAR T cell infusion [42]. A similar timeline (11 days) for the occurrence of cardiotoxicity is also reported in another singlecenter study [43].

The exact incidence of CRS-associated CV adverse outcomes is unknown. Although CRS is reported to occur fairly frequently (70-90\%), the toxic effects mediated by cytokine release are typically mild in severity. However, $10-20 \%$ of patients can develop life-threatening complications such as vascular leak syndrome with circulatory collapse and multiorgan failure $[8 \bullet \bullet, 12,42]$. In pivotal studies involving 287 patients, cardiac arrest was reported in 4 patients and cardiac failure in 2 patients, likely underestimating the true incidence and impact of cardiotoxicity associated with CAR T cell therapy [3-5].

A retrospective study examining CAR T cell cardiotoxicity in 137 patients found that $5.8 \%$ of patients developed cardiomyopathy in the context of grade $\geq 2$ CRS [12]. However, the incidence of cardiomyopathy is likely underestimated in this study since only 29 of 137 patients underwent serial echocardiography. Another single-center study of 145 patients reported that around $15 \%$ of patients developed clinical heart failure. However, the incidence of systolic dysfunction is not clear given only a small proportion of patients (17 of 145) had sequential echocardiogram available [43]. In our study of 187 patients undergoing CAR T cell therapy for refractory or relapsed, aggressive non-Hodgkin lymphoma, baseline echocardiogram was performed in all, and 116 patients with grade $\geq 2$ CRS had follow-up echocardiogram. We found that $10.3 \%$ of patients developed new or worsening cardiomyopathy, with a decline in median LVEF from 58 to $37 \%$ [42].

Several risk factors for the high-grade CRS development have been identified and include high disease burden, higher infused CAR T cell dose, high-intensity lymphodepletion regimen, preexisting endothelial activation, and severe thrombocytopenia [6]. However, the risk factors for the development of CAR $\mathrm{T}$ cell therapy-associated cardiotoxicity have not been well known. 
In our study of 187 patients, older age, high-grade CRS, hyperlipidemia, coronary artery disease, and the use of renin-angiotensin inhibitors and betablockers at baseline were associated with the development of new or worsening cardiomyopathy [42]. This study did not find any such association with prior anthracycline or radiation exposure or history of stem cell transplantation [42]. Similarly, another single-center study did not find any influence of cancer subtype (CLL, ALL, and DLBCL) on the development of MACE. However, the study interestingly found that the higher baseline creatinine was associated with MACE following CAR T cell therapy [43].

\section{Pre-CAR T cell therapy cardiovascular workup}

Serious cardiovascular events were reported in early clinical trials and practice despite highly selective patient enrollment without any significant active cardiovascular issues $[8 \bullet \bullet]$. Our study suggests that preexisting cardiovascular risk factors and disease, as well as older age, can further increase the risk of such adverse cardiovascular events [42]. This signifies that when CAR T cell therapy is used more broadly under real-world conditions in higher risk patients, the incidence and impact of such cardiotoxicity might be higher than estimated from early reports.

While there is no standardized protocol for pretherapy cardiovascular evaluation, we recommend detailed cardiovascular history taking and performance of ECG as well as echocardiogram for all patients. For patients with any significant cardiovascular symptoms, prior history of cardiovascular disease, impaired exercise capacity, or any abnormality noted on initial workup, further testing with imaging stress test may need to considered to rule out underlying occult coronary ischemia or other structural heart diseases to determine that the patient will be able to withstand the hemodynamic changes associated with CRS or not $[8 \bullet \bullet]$. Additionally, optimization and possibly downtitration or discontinuation of antihypertensive, antiplatelets, and anticoagulation agents should be considered given the elevated risk of hypotension and thrombocy-

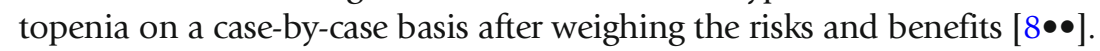

Given that the vast majority of cardiotoxicity occurs in the context of CRS [12, 42], and additionally, the hemodynamic collapse related to CRS may lead to MACE in patients with preexisting cardiovascular disease [42], prompt recognition of CRS is crucial. A high degree of suspicion, clinical surveillance, and ongoing cardiac monitoring with close monitoring of heart rate and blood pressure (BP) are required during the index hospitalization. While the markers of inflammation such as C-reactive protein, ferritin, and IL-6 are routinely checked and typically elevated with CRS, it is not specific and could be elevated in many other conditions.

While troponin elevation has been associated with CRS and adverse cardiovascular events [12], routine surveillance with troponin may not be required or helpful. A repeat echocardiogram for surveillance may be considered for patients with grade $\geq 2$ CRS or those who experience hemodynamic instability or cardiovascular symptoms [42].

While CRS and associated cardiotoxicity is most often the cause of hemodynamic instability in patients undergoing CAR T cell therapy, other possible 
etiologies, such as sepsis, tumor lysis syndrome, pulmonary embolism, or primary cardiac events, need to be considered and ruled out.

\section{Management}

While there is no formal guideline for the management of CAR T cell therapyassociated adverse cardiovascular events, our knowledge is evolving based on clinical practice. Supportive hemodynamic care and targeted anti-IL-6 therapy, as well as possibly broader immunosuppression with corticosteroids, are the cornerstones of the management.

Supportive care

If post-CAR T cell therapy, the patient develops clinically significant hypotension and tachycardia likely secondary to early CRS, intravenous fluid resuscitation should be considered weighing the risk of vascular leak and pulmonary congestion [6]. Vasopressors should be considered for persistent hypotension despite volume resuscitation.

Given that IL- 6 is thought to play a central role in CAR T cell therapyassociated CRS as well as myocardial depression [39], anti-IL-6 therapy is considered a first-line therapy for high-grade CRS [44]. Tocilizumab is a monoclonal anti-IL- 6 receptor antibody and is approved by the FDA as a first-line agent to be used for the management of CRS-related toxicity. Typically, $8 \mathrm{mg} / \mathrm{kg}$ intravenously is given, and this dose could be repeated up to 3 times $8 \mathrm{~h}$ apart [44]. A recent study demonstrates that a shorter time from CRS onset to tocilizumab was associated with a lower rate of adverse cardiovascular events. However, there is also an unproven concern that the efficacy of the CAR T cell therapy may be reduced with tocilizumab administration. Hence, there is a wide variation in practice, and some experts believe that it should be reserved for patients with hypoxia or hypotension requiring BP support for longer than $24 \mathrm{~h}$, or those with unstable arrhythmia, evidence of myocardial damage (increased troponin level), or new

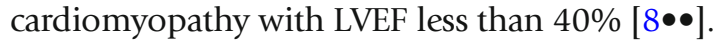

Siltuximab is another monoclonal antibody that blocks IL-6 signaling by binding to IL-6 itself and preventing it from activating immune effector cells [45]. While it is not examined extensively for use in the context of CRS management, and hence not FDA approved, it has a higher affinity for IL- 6 than tocilizumab and can be considered in patients not responding to tocilizumab and corticosteroids. As siltuximab binds directly to IL-6 and not its receptors, it is potentially better as it may decrease levels of IL- 6 in the central nervous system, whereas tocilizumab may potentially increase the systemic and possibly the central nervous system IL-6 level, which could precipitate or worsen neurotoxicity [46]. 
considered a second-line therapy for the management of CRS refractory to tocilizumab. Although exposure to corticosteroids is thought to potentially decrease CAR T cell efficacy, results from initial clinical trials did not show an association between the use of either and response rates [46].

The prognosis of patients who develop CAR T cell therapy-associated adverse cardiovascular events is not well understood. Our recent study shows that in patients who developed CAR T cell therapy-associated cardiomyopathy, LVEF recovered in $75 \%$ of patients with supportive care [42]. However, our knowledge is limited regarding the potential late or long-term cardiovascular effects of altering the immune system. Long-term follow-up studies would be required to understand if patients are at a higher risk of developing metabolic syndrome, hypertension, vascular disease, and cardiomyopathy after a latent period, following CAR T cell therapy due to the immune system modulation and continued circulation of CAR T cells.

\section{COVID-19 and immunotherapy for cancer}

Patients with cancer are typically at higher risk of infection for a variety of reasons, including the compromised immune system, as well as secondary to antineoplastic therapy, which may lead to leukopenia, disruption in barriers to infection, and shifts in microbial flora [47]. Additionally, patients with cancer are found to experience worse outcomes with COVID-19, especially in patients who had both preexisting cancer and cardiovascular disease [48].

COVID-19 may cause myocardial injury either due to direct viral myocardial invasion (myocarditis) or secondary to CRS resulting from hyperinflammatory response to COVID-19, angiotensin-converting enzyme-2 receptor modulation-mediated cardiac and endothelial injury or due to microvascular dysfunction and thrombosis [49]. While any antineoplastic therapy may compromise the patient's immune system and potentially worsen COVID-19 severity, immunotherapy-both ICI and CAR T cell therapy-present a complex problem as they just not can potentially exacerbate the severity of COVID-19 illness, but cardiovascular complications secondary to COVID-19 may mimic those of cardiotoxicity associated with ICI (myocarditis) and CAR T cell therapy (CRS-associated myocardial injury), and in turn can delay the diagnosis and appropriate management.

We recommend that all patients should be screened for active COVID-19 infection prior to initiating both ICI and CAR T cell therapy. Additionally, as the usual treatment of ICI-associated myocarditis consists of immunosuppression with high-dose corticosteroids which can potentially worsen the viral infection, in areas of high transmission, if patients undergoing ICI presents with any cardiovascular symptoms, differential diagnosis needs to include not just ICIassociated myocarditis, but also COVID-19-associated myocardial injury to guide the therapy. An anti-IL-6 agent such as tocilizumab has shown efficacy in the treatment of COVID-19-associated CRS as well, and hence, it is reasonable to have a low threshold for its use in the context of CRS for patients undergoing CAR T cell therapy given the potential of unrecognized coinfection with COVID-19 [50]. 


\section{Compliance with Ethical Standards}

\section{Conflict of interest}

Natalie Dal'bo, Rushin Patel, Rohan Parikh, Sachin P. Shah, Avirup Guha, Sourbha S. Dani, and Sarju Ganatra declare that they have no conflict of interest.

\section{Animal rights and informed consent}

This article does not contain any studies with human or animal subjects performed by any of the authors.

\section{References and Recommended Reading}

Papers of particular interest, published recently, have been highlighted as:

- Of major importance

1. Hodi FS, O'Day SJ, McDermott DF, Weber RW, Sosman JA, Haanen JB, et al. Improved survival with ipilimumab in patients with metastatic melanoma. $\mathrm{N}$ Engl J Med. 2010;363(8):711-23. https://doi.org/10. 1056/NEJMoa1003466.

2. Robert C, Thomas L, Bondarenko I, O'Day S, Weber J, Garbe C, et al. Ipilimumab plus dacarbazine for previously untreated metastatic melanoma. N Engl J Med. 2011;364(26):2517-26. https://doi.org/10.1056/ NEJMoa1104621.

3. Maude SL, Laetsch TW, Buechner J, Rives S, Boyer M, Bittencourt $\mathrm{H}$, et al. Tisagenlecleucel in children and young adults with B-cell lymphoblastic leukemia. N Engl J Med. 2018;378(5):439-48. https://doi.org/10. 1056/NEJMoa1709866.

4. Neelapu SS, Locke FL, Bartlett NL, Lekakis LJ, Miklos DB, Jacobson CA, et al. Axicabtagene ciloleucel CAR Tcell therapy in refractory large B-cell lymphoma. N Engl J Med. 2017;377(26):2531-44. https://doi.org/10. 1056/NEJMoa1707447.

5. Schuster SJ, Bishop MR, Tam CS, Waller EK, Borchmann P, McGuirk JP, et al. Tisagenlecleucel in adult relapsed or refractory diffuse large B-cell lymphoma. N Engl J Med. 2019;380(1):45-56. https://doi. org/10.1056/NEJMoa1804980.

6. Ganatra S, Parikh R, Neilan TG. Cardiotoxicity of immune therapy. Cardiol Clin. 2019;37(4):385-97. https://doi.org/10.1016/j.ccl.2019.07.008.

7. Ganatra S, Neilan TG. Immune checkpoint inhibitorassociated myocarditis. Oncologist. 2018;23:879-86. https://doi.org/10.1634/theoncologist.2018-0130.

8.• Ganatra S, Carver JR, Hayek SS, Ky B, Leja MJ, Lenihan DJ, et al. Chimeric antigen receptor T-cell therapy for cancer and heart: JACC Council perspectives. J Am Coll Cardiol. 2019;74(25):3153-63. https://doi.org/10. 1016/j.jacc.2019.10.049

This is an American College of Cardiology (ACC) CardioOncology council perspective on cardiovascular effects of CAR T-cell therapy with proposed pre-CAR T cell therapy workup and management strategies.
9. Postow MA, Sidlow R, Hellmann MD. Immune-related adverse events associated with immune checkpoint blockade. N Engl J Med. 2018;378(2):158-68. https:// doi.org/10.1056/NEJMra1703481.

10.• Mahmood SS, Fradley MG, Cohen JV, Nohria A, Reynolds KL, Heinzerling LM, et al. Myocarditis in patients treated with immune checkpoint inhibitors. J Am Coll Cardiol. 2018;71(16):1755-64. https://doi. org/10.1016/j.jacc.2018.02.037

This paper encompasses the data from international ICI-associated myocarditis registry and delineates risk factors, sensitivity, and specificity of various diagnostic tests and treatment strategies.

11. Maldini CR, Ellis GI, Riley JL. CAR T cells for infection, autoimmunity and allotransplantation. Nat Rev Immunol. 2018;18(10):605-16. https://doi.org/10. 1038/s41577-018-0042-2.

12. Alvi RM, Frigault MJ, Fradley MG, Jain MD, Mahmood SS, Awadalla M, et al. Cardiovascular events among adults treated with chimeric antigen receptor T-cells (CAR-T). J Am Coll Cardiol. 2019;74(25):3099-108. https://doi.org/10.1016/j.jacc.2019.10.038.

13. Johnson DB, Balko JM, Compton ML, Chalkias S, Gorham J, Xu Y, et al. Fulminant myocarditis with combination immune checkpoint blockade. N Engl J Med. 2016;375(18):1749-55. https://doi.org/10. 1056/NEJMoa1609214.

14. Love VA, Grabie N, Duramad P, Stavrakis G, Sharpe A, Lichtman A. CTLA-4 ablation and interleukin-12 driven differentiation synergistically augment cardiac pathogenicity of cytotoxic T lymphocytes. Circ Res. 2007;101(3):248-57. https://doi.org/10.1161/ CIRCRESAHA.106.147124.

15. Nishimura H, Okazaki T, Tanaka Y, Nakatani K, Hara M, Matsumori A, et al. Autoimmune dilated cardiomyopathy in PD-1 receptor-deficient mice. Science. 2001;291(5502):319-22. https://doi.org/10.1126/ science.291.5502.319.

16. Okazaki T, Tanaka Y, Nishio R, Mitsuiye T, Mizoguchi A, Wang J, et al. Autoantibodies against cardiac 
troponin I are responsible for dilated cardiomyopathy in PD-1-deficient mice. Nat Med. 2003;9(12):147783. https://doi.org/10.1038/nm955.

17. Grabie N, Gotsman I, DaCosta R, Pang H, Stavrakis G, Butte MJ, et al. Endothelial programmed death-1 ligand 1 (PD-L1) regulates CD8+ T-cell mediated injury in the heart. Circulation. 2007;116(18):2062-71. https://doi.org/10.1161/CIRCULATIONAHA.107. 709360.

18. Escudier M, Cautela J, Malissen N, Ancedy Y, Orabona $\mathrm{M}$, Pinto J, et al. Clinical features, management, and outcomes of immune checkpoint inhibitor-related cardiotoxicity. Circulation. 2017;136(21):2085-7. https://doi.org/10.1161/CIRCULATIONAHA.117. 030571 .

19. Sarocchi M, Grossi F, Arboscello E, Bellodi A, Genova C, Dal Bello MG, et al. Serial troponin for early detection of nivolumab cardiotoxicity in advanced nonsmall cell lung cancer patients. Oncologist. 2018;23(8):936-42. https://doi.org/10.1634/ theoncologist.2017-0452.

20. Läubli H, Balmelli C, Kaufmann L, Stanczak M, Syedbasha M, Vogt D, et al. Influenza vaccination of cancer patients during PD-1 blockade induces serological protection but may raise the risk for immunerelated adverse events. J Immunother Cancer. 2018;6(1):40. https://doi.org/10.1186/s40425-0180353-7.

21. Awadalla M, Golden DLA, Mahmood SS, Alvi RM, Mercaldo ND, Hassan MZO, et al. Influenza vaccination and myocarditis among patients receiving immune checkpoint inhibitors. J Immunother Cancer. 2019;7(1):53. https://doi.org/10.1186/s40425-0190535-y.

22. Lee C, Zafar A, Drobni ZD, et al. Pre-existing cardiovascular risk factors are associated with the development of immune checkpoint inhibitor myocarditis. AHA Abstract. 2020;Submitted.

23. Awadalla M, Mahmood SS, Groarke JD, Hassan MZO, Nohria A, Rokicki A, et al. Global longitudinal strain and cardiac events in patients with immune checkpoint inhibitor-related myocarditis. J Am Coll Cardiol. 2020;75(5):467-78. https://doi.org/10.1016/j.jacc. 2019.11.049.

24. Ferreira VM, Schulz-Menger J, Holmvang G, Kramer $\mathrm{CM}$, Carbone I, Sechtem U, et al. Cardiovascular magnetic resonance in nonischemic myocardial inflammation: expert recommendations. J Am Coll Cardiol. 2018;72(24):3158-76. https://doi.org/10.1016/j.jacc. 2018.09.072.

25. Zhang L, Awadalla M, Mahmood SS, Nohria A, Hassan $\mathrm{MZO}$, Thuny F, et al. Cardiovascular magnetic resonance in immune checkpoint inhibitor-associated myocarditis. Eur Heart J. 2020;41(18):1733-43. https://doi.org/10.1093/eurheartj/ehaa051.

26. Hauck AJ, Kearney DL, Edwards WD. Evaluation of postmortem endomyocardial biopsy specimens from 38 patients with lymphocytic myocarditis: implications for role of sampling error. Mayo Clin Proc. 1989;64(10):1235-45.

27. Maisch B, Portig I, Ristic A, Hufnagel G, Pankuweit S. Definition of inflammatory cardiomyopathy (myocarditis): on the way to consensus. A status report. Herz. 2000;25(3):200-9.

28.• Zhang L, Zlotoff DA, Awadalla M, Mahmood SS, Nohria A, Hassan MZO, et al. Major adverse cardiovascular events and the timing and dose of corticosteroids in immune checkpoint inhibitor-associated myocarditis. Circulation. 2020;141(24):2031-4. https://doi.org/10.1161/circulationaha.119.044703

This manuscript demonstrates the importance of prompt initiation of high-dose corticosteroids for the treatment of ICIassociated myocarditis.

29. Kwon HJ, Cote TR, Cuffe MS, Kramer JM, Braun MM. Case reports of heart failure after therapy with a tumor necrosis factor antagonist. Ann Intern Med. 2003;138(10):807-11.

30. Salem JE, Allenbach Y, Vozy A, Brechot N, Johnson DB, Moslehi JJ, et al. Abatacept for severe immune checkpoint inhibitor-associated myocarditis. N Engl J Med. 2019;380(24):2377-9. https://doi.org/10.1056/ NEJMc1901677.

31. Esfahani $\mathrm{K}$, Buhlaiga $\mathrm{N}$, Thébault $\mathrm{P}$, Lapointe R, Johnson NA, Miller WH. Alemtuzumab for immune-related myocarditis due to PD-1 therapy. N Engl J Med. 2019;380(24):2375-6. https://doi.org/10.1056/ NEJMc1903064.

32. Moslehi JJ, Salem JE, Sosman JA, Lebrun-Vignes B, Johnson DB. Increased reporting of fatal immune checkpoint inhibitor-associated myocarditis. Lancet. 2018;391(10124):933. https://doi.org/10.1016/ S0140-6736(18)30533-6.

33. Brahmer JR, Lacchetti C, Schneider BJ, Atkins MB, Brassil KJ, Caterino JM, et al. Management of immunerelated adverse events in patients treated with immune checkpoint inhibitor therapy: American Society of Clinical Oncology clinical practice guideline. J Clin Oncol. 2018;36(17):1714-68. https://doi.org/10. 1200/JCO.2017.77.6385.

34. Pollack MH, Betof A, Dearden H, Rapazzo K, Valentine I, Brohl AS, et al. Safety of resuming anti-PD-1 in patients with immune-related adverse events (irAEs) during combined anti-CTLA-4 and anti-PD1 in metastatic melanoma. Ann Oncol. 2018;29(1):250-5. https://doi.org/10.1093/annonc/mdx642.

35. Sadelain M, Brentjens R, Riviere I. The basic principles of chimeric antigen receptor design. Cancer Discov. 2013;3(4):388-98. https://doi.org/10.1158/21598290.CD-12-0548.

36. June $\mathrm{CH}$, Sadelain $\mathrm{M}$. Chimeric antigen receptor therapy. N Engl J Med. 2018;379(1):64-73. https://doi. org/10.1056/NEJMra1706169.

37. Raje N, Berdeja J, Lin Y, Siegel D, Jagannath S, Madduri $\mathrm{D}$, et al. Anti-BCMA CAR T-cell therapy bb2121 in relapsed or refractory multiple myeloma. $\mathrm{N}$ Engl J Med. 2019;380(18):1726-37. https://doi.org/10.1056/ NEJMoa1817226. 
38. Burstein DS, Maude S, Grupp S, Griffis H, Rossano J, Lin K. Cardiac profile of chimeric antigen receptor $\mathrm{T}$ cell therapy in children: a single-institution experience. Biol Blood Marrow Transplant. 2018;24(8):1590-5. https://doi.org/10.1016/j.bbmt.2018.05.014.

39. Pathan N, Hemingway CA, Alizadeh AA, Stephens AC, Boldrick JC, Oragui EE, et al. Role of interleukin 6 in myocardial dysfunction of meningococcal septic shock. Lancet. 2004;363(9404):203-9. https://doi.org/ 10.1016/S0140-6736(03)15326-3.

40. Cameron BJ, Gerry AB, Dukes J, Harper JV, Kannan V, Bianchi FC, et al. Identification of a Titin-derived HLAA1-presented peptide as a cross-reactive target for engineered MAGE A3-directed T cells. Sci Transl Med. 2013;5(197):197ra03. https://doi.org/10.1126/ scitranslmed.3006034.

41. Hay KA, Hanafi LA, Li D, Gust J, Liles WC, Wurfel MM, et al. Kinetics and biomarkers of severe cytokine release syndrome after CD19 chimeric antigen receptormodified T-cell therapy. Blood. 2017;130(21):2295306. https://doi.org/10.1182/blood-2017-06-793141.

42. Ganatra S, Redd R, Hayek SS, Parikh R, Azam T, Yanik $\mathrm{G}$, et al. Chimeric antigen receptor T-cell therapy associated cardiomyopathy in patients with refractory or relapsed non-Hodgkin lymphoma. Circulation. 2020;In Press.

43. Lefebvre B, Kang Y, Smith AM, Frey NV, Carver JR, Scherrer-Crosbie M. Cardiovascular effects of CAR T cell therapy. A retrospective study. JACC: CardioOncol. 2020;2(2):193-203. https://doi.org/10.1016/j.jaccao. 2020.04.012.

44. Le RQ, Li L, Yuan W, Shord SS, Nie L, Habtemariam BA, et al. FDA approval summary: tocilizumab for treatment of chimeric antigen receptor $T$ cell-induced severe or life-threatening cytokine release syndrome. Oncologist. 2018;23(8):943-7. https://doi.org/10.1634/ theoncologist.2018-0028.

45. Riegler LL, Jones GP, Lee DW. Current approaches in the grading and management of cytokine release syndrome after chimeric antigen receptor T-cell therapy. Ther Clin Risk Manag. 2019;15:323-35. https:// doi.org/10.2147/TCRM.S150524.

46. Locke FL, Neelapu SS, Bartlett NL, Lekakis LJ, Jacobson CA, Braunschweig I, et al. Preliminary results of prophylactic tocilizumab after axicabtageneciloleucel (axicel; KTE-C19) treatment for patients with refractory, aggressive non-Hodgkin lymphoma (NHL). Blood. 2017;130(Suppl 1):1547.

47. Ganatra S, Hammond SP, Nohria A. The novel coronavirus disease (COVID-19) threat for patients with cardiovascular disease and cancer. JACC: CardioOncol. 2020;80:350-5. https://doi.org/10.1016/j.jaccao. 2020.03.001.

48. Ganatra S, Dani S, Redd R, Rieger-Christ K, Patel R, Parikh R, et al. Outcomes of the novel coronavirus disease 2019 in patients with a history of cancer and co-morbid cardiovascular disease. J Natl Compr Canc Netw. 2020;In Press.

49. Ganatra S, Dani SS, Shah S, Asnani A, Neilan TG, Lenihan D, et al. Management of cardiovascular disease during coronavirus disease (COVID-19) pandemic. Trends Cardiovasc Med. 2020;30:315-25. https://doi.org/10.1016/j.tcm.2020.05.004.

50. Lenihan D, Carver J, Porter C, Liu JE, Dent S, Thavendiranathan P et al. Cardio-oncology care in the era of the coronavirus (COVID-19) pandemic: an International Cardio-Oncology Society (ICOS) statement on cardiac safety in a new treatment paradigm. CA Cancer J Clin. 2020;In Press.

\section{Publisher's Note}

Springer Nature remains neutral with regard to jurisdictional claims in published maps and institutional affiliations. 